\title{
Sciendo
}

Int. J. of Applied Mechanics and Engineering, 2019, vol.24, No.3, pp.527-538

DOI: 10.2478/ijame-2019-0033

\section{SIMULTANEOUS CHEMICAL REACTIONS EFFECT ON DISPERSION OF A SOLUTE IN PERISTALTIC PROPULSION OF A NEWTONIAN FLUID IN AN INCLINED CHANNEL WITH WALL PROPERTIES}

\author{
M.Y. DHANGE ${ }^{*}$ and G.C. SANKAD \\ Department of Mathematics \\ (Affiliated to Visvesvaraya Technological University, Belagavi, India) \\ B.L.D.E.A.'S.V.P.Dr.P.G. Halakatti College of Engineering and Technology \\ Vijayapur (586103), Karnataka, INDIA \\ E-mails: "math.mallinath@bldeacet.ac.in, math.gurunath@bldeacet.ac.in
}

\begin{abstract}
In this paper, the dispersion of a solute in the peristaltic propulsion of an incompressible and viscous fluid through a permeable medium under the influence of wall properties with simultaneous homogeneous, heterogeneous chemical reactions in an inclined uniform channel has been studied. The issue is studied through conditions of Taylor's limit and long wavelength hypothesis. The mean effective coefficient of scattering expression is computed and outcomes are interpreted physically through graphs.
\end{abstract}

Key words: dispersion, inclined channel, viscous fluid, peristaltic propulsion, wall properties.

\section{Introduction}

"Peristaltikos" is a Greek word which implies clasping and compressing, from which the word 'peristaltic' is derived. Peristalsis is a coordinated response wherein a wave of contraction preceded by a wave of relaxation passes down a hollow viscus. Thus 'peristalsis' is the rhythmic sequence of smooth muscle contractions that progressively squeeze one small section of the tract and then the next to push the content along the tract. As they are propelled along, they would always enter a segment which had actively relaxed and enlarged to receive them. From the perspective of fluid dynamics, peristalsis is typified by the dynamical interface of the fluid flow and movement of the flexible boundaries of the conduit. This technique is also applied in several biomedical strategies; e.g., finger and roller thrusts. In view of its significance, numerous specialists have inspected the creeping sinusoidal flow of diverse fluids under many conditions [16]. In physiological structures, it is known that all vessels are not straight but have some inclination with the axis. The gravitational strength is accounted for due to the consideration of an inclined channel. A few scholars have studied the peristaltic stream of Newtonian and non-Newtonian liquids in an inclined conduit with various conditions [7-9]. After this study, some investigators have studied the wall effects on different fluids with peristalsis [10-17].

Dispersion is the process by which a material is transported from one portion of a system to another as a result of random molecular motion. Taylor [18] explored the incompressible and viscous laminar stream of a liquid in a round tube with a scattering of a solute material. Aris [19], Padma-Rao [20], Gupta and Gupta [21], Sobh [22], Chandra-Philip [23], and Dutta et al. [24] considered the scattering of a solute material in a viscous liquid under distinct situations. These studies have been extended to non-Newtonian liquids by many experts [25-27].

A porous medium is formed by lots of relatively thoroughly packed particles or solid matrix with its void filled with liquids. A porous medium is a material containing pores or spaces in between the solid

\footnotetext{
* To whom correspondence should be addressed
} 
matter through which gas or liquid can pass. The bile duct, gall bladder with stones and human lungs, limestone, beach sand, sandstone, are some of the examples of natural porous media. Moreover, movements of underground water, liquid filtration and water discharge in river beds are examples of flow through a permeable medium. The study of flow in permeable media has a vital role in understanding the transportation process in kidneys, gallbladder with stones and lungs. Most of the tissues in the body are deformable permeable media. The appropriate functioning of the tissues depends on the stream of blood, nutrients and other substances.

Peristalsis, permeability, and diffusion are most essential characteristics in bio-medical, natural and chemical processes. Existing information on the topic shows that an analytical treatment of creeping sinusoidal flow and dispersion of an incompressible and viscous liquid in an inclined channel through a permeable medium with chemical reactions and wall features has been never reported. Motivated by this fact, we have investigated the chemical and wall feature effects on the creeping sinusoidal stream and dispersion in an incompressible and viscous fluid flow through a permeable medium in an inclined channel. Blood vessels are part of the circulatory system, through which nutrients, blood, hormones, and other important substances easily pass (Lightfoot [29]). The core outcomes are investigated for different values of penetrating constraints through graphs and presented in the conclusions.

\section{Mathematical model}

Consider the peristaltic stream of an incompressible and viscous liquid in an inclined porous channel. Figure 1 shows the wave shape of the peristaltic wave.

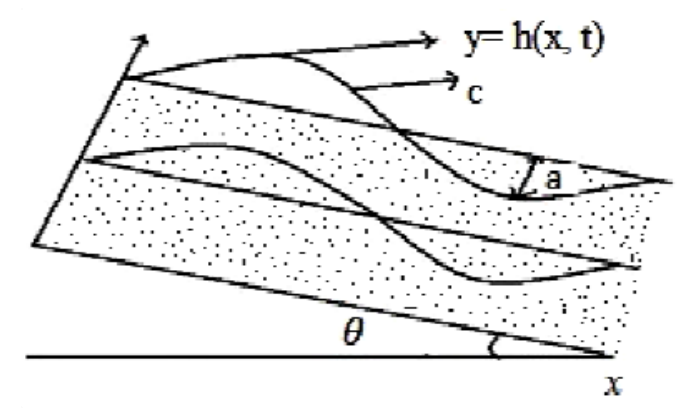

Fig.1. Geometry of the model.

The wave shape is assumed as

$$
y= \pm\left[a \sin \frac{2 \pi}{\lambda}(x-c t)+d\right]= \pm \mathfrak{h},
$$

where $a$ is the amplitude, $\lambda$ is the wavelength of the wave, and $d$ is the half width of the channel.

The equivalent stream equations (Gupta-Seshadri [28]) of the current issue are

$$
\begin{aligned}
& \frac{\partial v}{\partial y}+\frac{\partial \mathcal{U}}{\partial x}=0 \\
& -\frac{\partial p}{\partial x}+\mu\left(\frac{\partial^{2}}{\partial x^{2}}+\frac{\partial^{2}}{\partial y^{2}}\right) \mathcal{U}-\frac{\mu}{\bar{k}} \mathcal{U}+\rho g \sin \theta=\rho\left[\frac{\partial}{\partial t}+\mathcal{U} \frac{\partial}{\partial x}+v \frac{\partial}{\partial y}\right] \mathcal{U},
\end{aligned}
$$




$$
-\frac{\partial p}{\partial y}+\mu\left(\frac{\partial^{2}}{\partial x^{2}}+\frac{\partial^{2}}{\partial y^{2}}\right) v-\frac{\mu}{\bar{k}} v-\rho g \cos \theta=\rho\left[\frac{\partial}{\partial t}+\mathcal{U} \frac{\partial}{\partial x}+v \frac{\partial}{\partial y}\right] v
$$

where $\mathcal{U}$ is the velocity component in the $x$-direction, $v$ is the velocity component in the $y$-direction, $\rho$ is the density of the fluid, $g$ is the gravity due to acceleration, $\theta$ is the angle of inclination, $p$ is the pressure, $\bar{k}$ is the permeable constraint and $\mu$ is the viscosity coefficient.

The condition of the flexible wall movement (Mittra-Prasad [10]) is specified as

$$
p-p_{0}=L(\hbar)
$$

where $L$ is the movement of the stretched membrane by the damping force and is given by the subsequent equation

$$
L=-T \frac{\partial^{2}}{\partial x^{2}}+m \frac{\partial^{2}}{\partial t^{2}}+C \frac{\partial}{\partial t}
$$

Here, $m$ is mass per unit area, $T$ is the membrane tension, and $C$ is the coefficient of viscous damping force.

Following Alemayehu-Radhakrishnamacharya [25] and using the long wavelength hypothesis, Eqs (2.2) to (2.4) yield

$$
\begin{aligned}
& \frac{\partial v}{\partial y}+\frac{\partial \mathcal{U}}{\partial x}=0, \\
& \mu \frac{\partial^{2} \mathcal{U}}{\partial y^{2}}-\frac{\mu}{\bar{k}} \mathcal{U}+\rho g \sin \theta-\frac{\partial p}{\partial x}=0, \\
& -\frac{\partial p}{\partial y}=0 .
\end{aligned}
$$

The associated periphery clauses are specified as

$$
\mathcal{U}=0, \quad \text { at } y= \pm \mathfrak{h} \text {. }
$$

It is assumed that $p_{0}=0$ and the channel walls are inextensible; hence, the horizontal displacement of the wall is zero and simply lateral movement takes place.

$$
\frac{\partial}{\partial x} L(\mathfrak{h})=\mu \frac{\partial^{2} \mathcal{U}}{\partial y^{2}}-\frac{\mu}{\bar{k}} \mathcal{U}+\rho g \sin \theta, \quad \text { at } \quad y= \pm \mathfrak{h}
$$

where

$$
\frac{\partial}{\partial x} L(\mathfrak{h})=\frac{\partial p}{\partial x}=-T \frac{\partial^{3} \mathfrak{h}}{\partial x^{3}}+m \frac{\partial^{3} \mathfrak{h}}{\partial x \partial t^{2}}+C \frac{\partial^{2} \mathfrak{h}}{\partial x \partial t}
$$

From Eqs (2.8) - (2.11), we get 


$$
\mathcal{U}(y)=\frac{1}{\mu\left(M^{\prime}\right)^{2}} A^{\prime}\left[\frac{\cosh \left(M^{\prime} y\right)}{\cosh \left(M^{\prime} \hbar\right)}-1\right]
$$

The mean speed is specified as

$$
\overline{\mathcal{U}}=\frac{1}{2 \mathfrak{h}} \int_{-\mathfrak{h}}^{\mathfrak{h}} \mathcal{U}(y) d y=\frac{1}{\mu\left(M^{\prime}\right)^{2}} A^{\prime}\left[\frac{\sinh \left(M^{\prime} \mathfrak{h}\right)}{M^{\prime} \mathfrak{h} \cosh \left(M^{\prime} \mathfrak{h}\right)}-1\right]
$$

Utilizing [25], the fluid speed is specified as

$$
\mathcal{U}_{x}=\mathcal{U}-\overline{\mathcal{U}}=\frac{1}{\mu\left(\mathbb{M}^{\prime}\right)^{2}} A^{\prime}\left[T_{1}^{\prime} \cosh \left(\mathbb{M}^{\prime} y\right)-T_{2}^{\prime}\right]
$$

where

$$
\begin{aligned}
& T_{1}^{\prime}=\frac{1}{\cosh \left(M^{\prime} \mathfrak{h}\right)}, \quad T_{2}^{\prime}=\frac{\sinh \left(M^{\prime} \mathfrak{h}\right)}{M^{\prime} \mathfrak{h} \cosh \left(M^{\prime} \mathfrak{h}\right)}, \quad A^{\prime}=\left(\frac{\partial p}{\partial x}-\frac{\rho g}{\mu} \sin \theta\right), \\
& P^{\prime}=\frac{\partial p}{\partial x}=m \frac{\partial^{3} \mathfrak{h}}{\partial x \partial t^{2}}+C \frac{\partial^{2} \mathfrak{h}}{\partial x \partial t}-T \frac{\partial^{3} \mathfrak{h}}{\partial x^{3}}, \quad M^{\prime}=\sqrt{\frac{1}{\bar{k}}} .
\end{aligned}
$$

\subsection{Diffusion with simultaneous homogeneous and heterogeneous chemical reactions}

Following Taylor [18] and Gupta-Gupta [21], the diffusing equation for the concentration $\mathbb{C}$ of the substance for the present issue under isothermal conditions is

$$
D \frac{\partial^{2} \mathbb{C}}{\partial y^{2}}-k_{l} \mathbb{C}=\frac{\partial \mathbb{C}}{\partial t}+\mathcal{U} \frac{\partial \mathbb{C}}{\partial x}
$$

Here $\mathbb{C}$ is the concentration of the fluid, $D$ is the diffusion coefficient for chemical responses, and $k_{1}$ is the rate constant of a chemical response.

For the standard estimations of physiologically basic parameters of this issue, it is typical that $\overline{\mathcal{U}} \approx \mathbb{C}$ ([25]).

Utilizing $\overline{\mathcal{U}} \approx \mathbb{C}$ of [25], and consequent dimensionless quantities

$$
\theta=\frac{t}{\bar{t}}, \quad \bar{t}=\frac{\lambda}{\overline{\mathcal{U}}}, \eta=\frac{y}{d}, \quad \xi=\frac{(x-\overline{\mathcal{U}} t)}{\lambda}, \mathbb{H}=\frac{\mathfrak{h}}{d}, P=\frac{d^{2}}{\mu C \lambda} P^{\prime}, \quad k=\frac{\bar{k}}{d^{2}}, \quad \gamma=\frac{\rho g}{\mu} .
$$

Equations (2.12), (2.15) and (2.16) yield

$$
-\varepsilon\left[(2 \pi)^{3}\left(E_{1}+E_{2}\right) \cos (2 \pi \xi)-(2 \pi)^{2} E_{3} \sin (2 \pi \xi)\right]=P,
$$




$$
\begin{aligned}
& \mathcal{U}_{x}=\frac{d^{2}}{\mu \mathcal{M}^{2}} A\left[T_{1} \cosh (\mathcal{M} \eta)-T_{2}\right] \\
& \frac{\partial^{2} \mathbb{C}}{\partial \eta^{2}}-\frac{k_{1} d^{2}}{D} \mathbb{C}=\frac{d^{2}}{\lambda D} \mathcal{U}_{x} \frac{\partial \mathbb{C}}{\partial \xi}
\end{aligned}
$$

where $\quad A=P-\gamma \sin \theta, T_{1}=\frac{1}{\cosh (\mathcal{M} \mathbb{H})}, T_{2}=\frac{\sinh (\mathcal{M} \mathbb{H})}{(\mathcal{M} \mathbb{H}) \cosh (\mathcal{M} \mathbb{H})}, \quad E_{l}\left(=-\frac{T d^{3}}{\lambda^{3} \mu C}\right) \quad$ is $\quad$ the rigidity, $E_{2}\left(=\frac{m C d^{3}}{\lambda^{3} \mu}\right)$ is the stiffness, $E_{3}\left(=\frac{C d^{3}}{\mu \lambda^{2}}\right)$ is the damping characteristic of the wall and $\varepsilon\left(=\frac{a}{d}\right)$ is the amplitude ratio.

The scattering with first-order irreversible chemical response taking place in the mass of the fluid medium and at the walls of the channel and walls are catalytic to the chemical response.

Hence, the boundary conditions at the walls (Chandra-Philip [23]) are given as

$$
\begin{aligned}
& 0=\frac{\partial \mathbb{C}}{\partial y}+\mathbb{F} \mathbb{C}, \quad \text { at } \quad y=\left[a \sin \frac{2 \pi}{\lambda}(x-\overline{\mathcal{U}} t)+d\right]=\mathfrak{h}, \\
& 0=\frac{\partial \mathbb{C}}{\partial y}-\mathbb{F} \mathbb{C}, \quad \text { at } \quad y=-\left[a \sin \frac{2 \pi}{\lambda}(x-\overline{\mathcal{U}} t)+d\right]=-\mathfrak{h} .
\end{aligned}
$$

From Eqs (2.17), (2.21) and (2.22), we get

$$
\begin{aligned}
& 0=\frac{\partial \mathbb{C}}{\partial \eta}+\beta \mathbb{C}, \quad \text { at } \quad \eta=[\varepsilon \sin (2 \pi \xi)+1]=\mathbb{H}, \\
& 0=\frac{\partial \mathbb{C}}{\partial \eta}-\beta \mathbb{C}, \quad \text { at } \quad \eta=-[\varepsilon \sin (2 \pi \xi)+1]=-\mathbb{H}
\end{aligned}
$$

where $\beta=\mathbb{F} d$ is the heterogeneous response rate compared to the catalytic response at the wall.

From Eqs (2.23) and (2.24), the solution of Eq.(2.22) it follows

$$
\mathbb{C}(\eta)=\frac{d^{4}}{\mu D \lambda \mathcal{M}^{2}} A \frac{\partial C}{\partial \xi}\left[T_{4} \cosh (\mathcal{M} \eta)-T_{5} \cosh (\alpha \eta)+T_{6}-T_{7} \cosh (\alpha \eta)\right],
$$

where $\quad \alpha=\sqrt{\frac{k_{1}}{D}} d, \quad \mathcal{M}=\mathbb{M}^{\prime} d=\sqrt{\frac{1}{k}}$.

The volumetric rate $Q$ is described as the rate in which the solute is pumped across a section of the channel for each unit breadth.

$$
\int_{-\mathbb{H}}^{\mathbb{H}} \mathbb{C} \mathcal{U}_{x} d \eta=\mathcal{Q}
$$


Using Eqs (2.19) and (2.25) in Eq (2.26), we obtain

$$
\mathcal{Q}=-2 \frac{d^{6}}{\lambda D \mu^{2}}\left(\frac{\partial \mathbb{C}}{\partial \xi}\right) G\left(\xi, \alpha, \beta, \varepsilon, E_{1}, E_{2}, E_{3}, k, \gamma, \theta\right),
$$

where

$$
\begin{aligned}
& G=\left[-\frac{A^{2}}{\mathcal{M}^{4}}\left(\begin{array}{l}
\frac{T_{1} T_{4}}{2} T_{8}-\left(T_{1} T_{5}+T_{1} T_{7}\right) T_{9}+\left(T_{1} T_{6}-T_{2} T_{4}\right) T_{10}+ \\
+\left(T_{2} T_{5}+T_{2} T_{7}\right) T_{11}-T_{2} T_{6} H
\end{array}\right)\right] \\
& T_{1}=\frac{1}{\cosh (\mathcal{M} \mathbb{H})}, T_{2}=\frac{\sinh (\mathcal{M} \mathbb{H})}{(\mathcal{M} \mathbb{H}) \cosh (\mathcal{M} \mathbb{H})}, T_{3}=\frac{\sinh (\mathcal{M} \mathbb{H})}{\alpha \sinh (\alpha \mathbb{H})}, T_{4}=\frac{1}{\left(\mathcal{M}^{2}-\alpha^{2}\right) \cosh (\mathcal{M} \mathbb{H})} \\
& T_{5}=\frac{(\mathcal{M} \sinh (\mathcal{M} \mathbb{H})+\beta \cosh (\mathcal{M} \mathbb{H})}{\left(\mathcal{M}^{2}-\alpha^{2}\right) \cosh (\mathcal{M} \mathbb{H})(\alpha \sinh (\alpha \mathbb{H})+\beta \cosh (\alpha \mathbb{H}))}, \quad T_{6}=\frac{\sinh (\mathcal{M} \mathbb{H})}{(\mathcal{M} \mathbb{H}) \alpha^{2} \cosh (\mathcal{M} \mathbb{H})}, \\
& T_{7}=\frac{\beta \sinh (\mathcal{M} \mathbb{H})}{(\mathcal{M} \mathbb{H}) \alpha^{2} \cosh (\mathcal{M} \mathbb{H})(\alpha \sinh (\alpha \mathbb{H})+\beta \cosh (\alpha \mathbb{H}))}, \quad T_{8}=\frac{2 \mathcal{M} \mathbb{H}+\sinh (2 \mathcal{M} \mathbb{H})}{2 \mathcal{M}}, \\
& T_{9}=\frac{(\mathcal{M} \sinh (\mathcal{M} \mathbb{H}) \cosh (\alpha \mathbb{H})-\alpha \cosh (\mathcal{M} \mathbb{H}) \sinh (\alpha \mathbb{H}))}{\left(\mathcal{M}^{2}-\alpha^{2}\right)}, T_{10}=\frac{\sinh (\mathcal{M} \mathbb{H})}{\mathcal{M}} \\
& T_{11}=\frac{\sinh (\alpha \mathbb{H})}{\alpha}
\end{aligned}
$$

Looking at Eq.(2.28) with Fick's law of dispersion, the scattering coefficient $D^{*}$ was computed such that the solute diffuses relatively to the plane moving with the typical speed of the stream and is specified as

$$
D^{*}=2 \frac{d^{6}}{D \mu^{2}} G\left(\xi, \alpha, \beta, \varepsilon, E_{1}, E_{2}, E_{3}, k, \gamma, \theta\right) .
$$

Let $\bar{G}$ be the mean of $G$, and is attained by the following equation

$$
\bar{G}=\int_{0}^{l} G\left(\xi, \alpha, \beta, \varepsilon, E_{1}, E_{2}, E_{3}, k, \gamma, \theta\right) d \xi .
$$

\section{Results and discussion}

The mean effective scattering coefficient is observed throughout the function $\bar{G}$ for simultaneous homogeneous, heterogeneous chemical reactions given by Eq.(2.30). Computational results have been 
generated by using the MATHEMATICA software and the end results are presented through graphs. It should be taken car that $E_{1}, E_{2}$, and $E_{3}$ cannot be zero altogether.
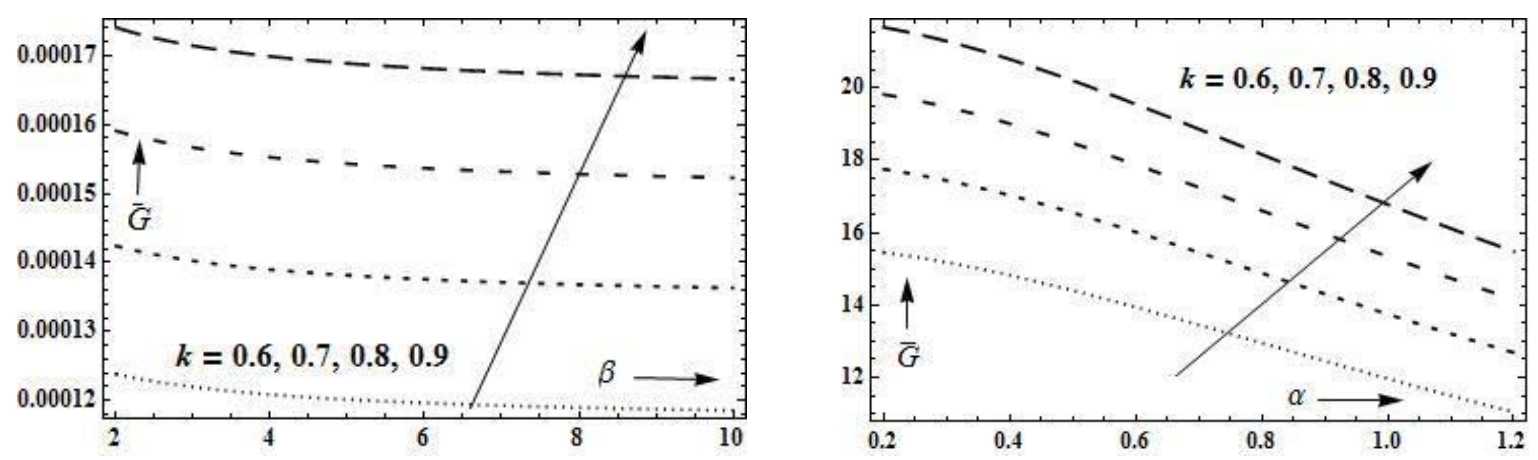

Fig.2. Illustration of $\bar{G}$ for $k$ with $\varepsilon=0.2$, Fig.3. Illustration of $\bar{G}$ for $k$ with $\varepsilon=0.2$,

$\alpha=1.0, \gamma=6.0, \theta=\pi / 6, E_{1}=0.1$,

$E_{2}=0.0, E_{3}=0.06$.

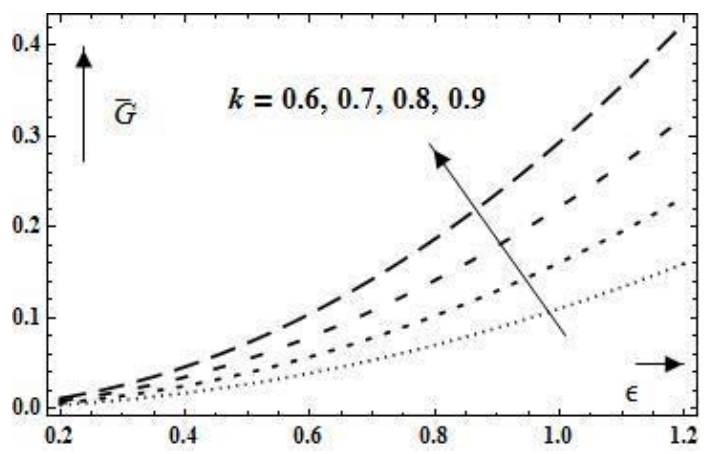

Fig.4. Illustration of $\bar{G}$ for $k$ with $\beta=5.0$, $\alpha=1.0, \gamma=6.0, \theta=\pi / 6, E_{1}=0.1$,
$E_{2}=4.0, E_{3}=0.0$.

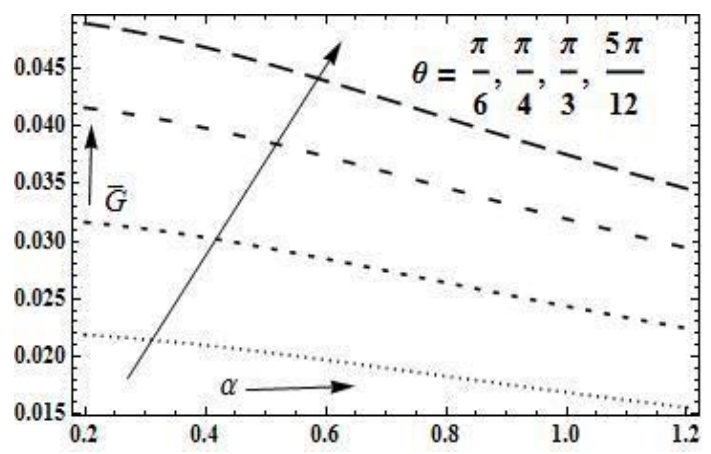

Fig.6. Illustration of $\bar{G}$ for $\theta$ with $\varepsilon=0.2$, $\beta=5.0, k=0.9, \gamma=6.0, E_{1}=0.1$, $E_{2}=0.0, E_{3}=0.06$.

$$
\beta=5.0, \gamma=6.0, \theta=\pi / 6, E_{1}=0.1 \text {, }
$$$$
E_{2}=4.0, E_{3}=0.06 \text {. }
$$

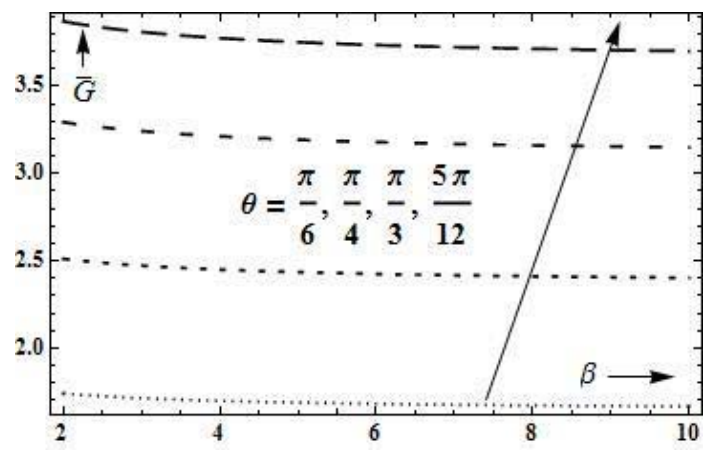

Fig.5. Illustration of $\bar{G}$ for $\theta$ with $\varepsilon=0.2$, $\alpha=1.0, k=0.9, \gamma=6.0, E_{1}=0.1$, $E_{2}=0.0, E_{3}=0.06$.

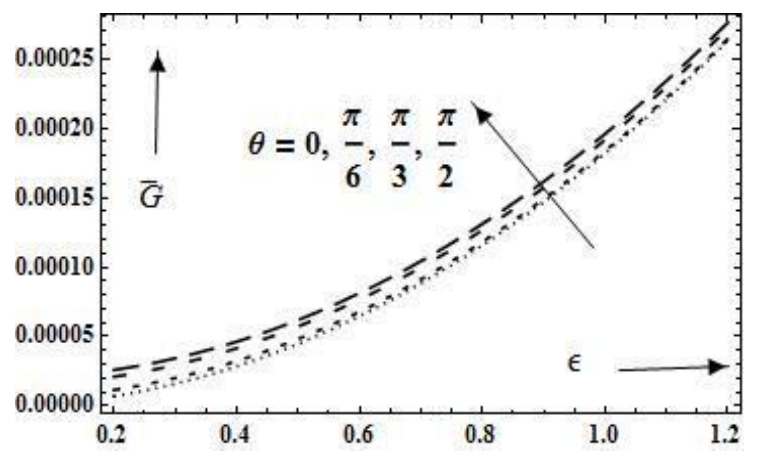

Fig.7. Illustration of $\bar{G}$ for $\theta$ with $\alpha=1.0$, $\beta=5.0, k=0.9, \gamma=6.0, E_{1}=0.1$, $E_{2}=0.0, E_{3}=0.06$. 

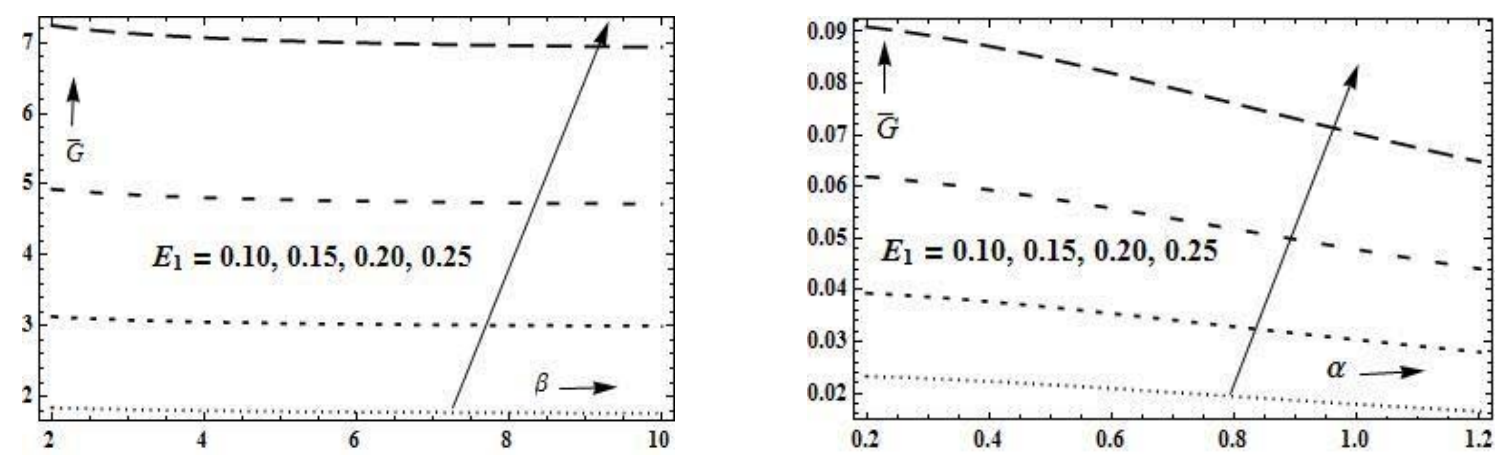

Fig.8. Illustration of $\bar{G}$ for $E_{1}$ with $\varepsilon=0.2$, Fig.9. Illustration of $\bar{G}$ for $E_{1}$ with $\varepsilon=0.2$, $\alpha=1.0, k=0.9, \gamma=6.0, \theta=\pi / 6$, $E_{2}=0.0, E_{3}=0.00$.

$$
\beta=5.0, k=0.9, \gamma=6.0, \theta=\pi / 6 \text {, }
$$
$E_{2}=0.0, E_{3}=0.06$.
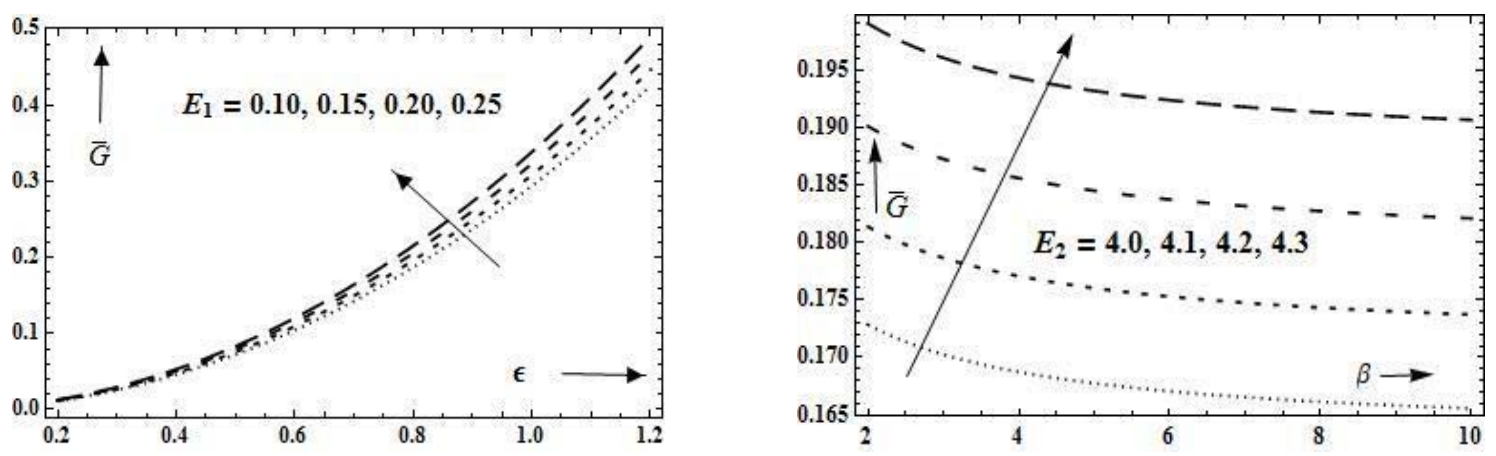

Fig.10. Illustration of $\bar{G}$ for $E_{1}$ with $\alpha=1.0$, Fig.11. Illustration of $\bar{G}$ for $E_{2}$ with $\varepsilon=0.2$, $\beta=5.0, k=0.9, \gamma=6.0, \quad \theta=\pi / 6$,
$E_{2}=4.0, E_{3}=0.00$. $\alpha=1.0, k=0.9, \gamma=6.0, \theta=\pi / 6, E_{1}=0.1$, $E_{3}=0.00$.
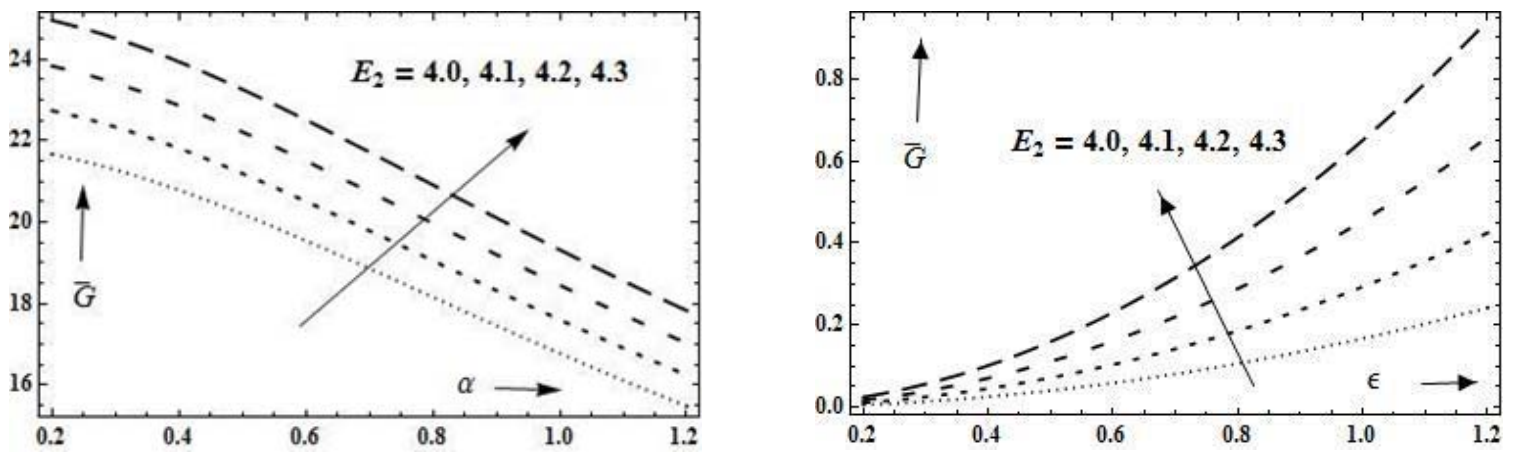

Fig.12. Illustration of $\bar{G}$ for $E_{2}$ with $\varepsilon=0.2$, Fig.13. Illustration of $\bar{G}$ for $E_{2}$ with $\beta=5.0$, $\beta=5.0, k=0.9, \gamma=6.0, \quad \theta=\pi / 6$, $E_{1}=0.1, E_{3}=0.06$.

$$
\begin{aligned}
& \alpha=1.0, k=0.9, \gamma=6.0, \theta=\frac{\pi}{6}, \\
& E_{1}=0.1, E_{3}=0.06
\end{aligned}
$$



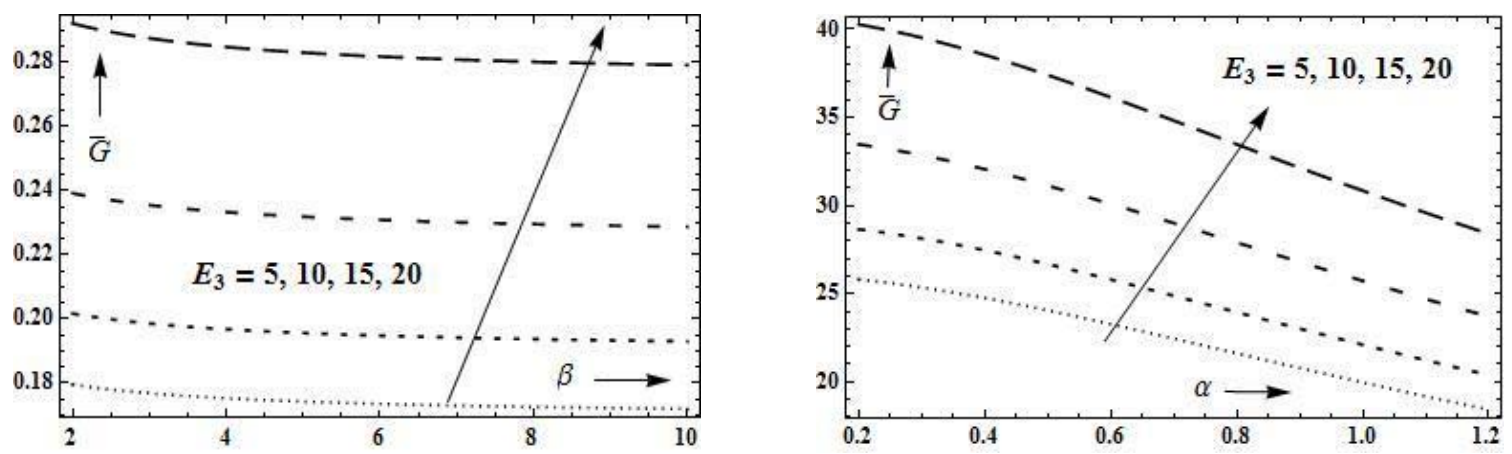

Fig.14. Illustration of $\bar{G}$ for $E_{3}$ with $\varepsilon=0.2$, Fig.15. Illustration of $\bar{G}$ for $E_{3}$ with $\varepsilon=0.2$, $\alpha=1.0, k=0.9, \gamma=6.0, \theta=\pi / 6, E_{1}=0.1, \quad \beta=5.0, k=0.9, \gamma=6.0, \theta=\pi / 6, E_{1}=0.1$, $E_{2}=4.0$. $E_{2}=4.0$.

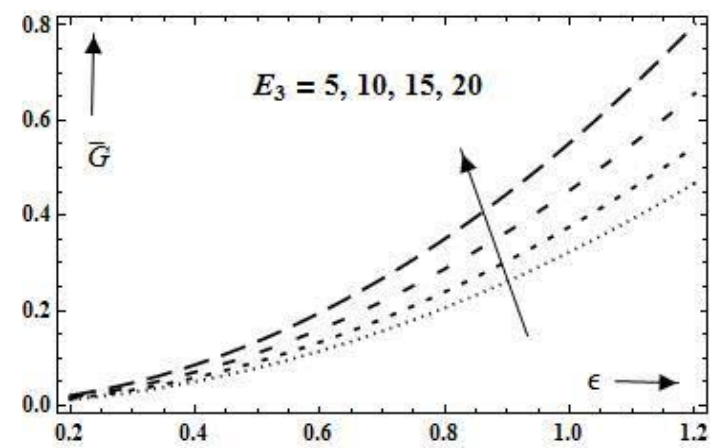

Fig.16. Illustration of $\bar{G}$ for $E_{3}$ with $\beta=5, \alpha=1.0, k=0.9, \gamma=6.0, \theta=\pi / 6, E_{1}=0.1, E_{2}=4.0$.

It is observed from Figs 2-4 that $\bar{G}$ rises with an increase in the permeability constraint $(k)$. It is also observed from Figs 5-7 that $\bar{G}$ grows as an angle of proclivity $(\theta)$ increases. These effects are in agreement with the results of Sankad-Radhakrishnamacharya [13].

From Figs $8-16$, it follows that $\bar{G}$ grows with a rise in the rigidity constraint of the wall $\left(E_{1}\right)$ toughness of the wall $\left(E_{2}\right)$ and viscous damping force of the wall $\left(E_{3}\right)$. This result is concurrent with the result of Hayat et al. [26], Ravikiran-Radhakrishnamacharya [27]. Furthermore, $\bar{G}$ ascends with an increment in the amplitude ratio $(\varepsilon)$ (Figs 4, 7, 10, 13, and 16). This result is in agreement with that of Sobh [22] and Alemayehu-Radhakrishnamacharya [25].

It is seen that $\bar{G}$ descends with an increase in the homogeneous chemical response rate $(\alpha)$ (Figs 3, $6,9,12$, and 15) and heterogeneous chemical response rate $(\beta)$ (Figs 2, 5, 8, 11, and 14). This result is common since a growth in $\alpha$ stimulates expansion in the sum of moles of solute experiences chemical response. These results agree with the arguments of Padma-Rao [20] and Hayat et al. [26].

\section{Conclusions}

The present study investigated the effect of chemical responses and wall properties on an incompressible and viscous fluid with creeping sinusoidal flow. The key results of this article are given below. 
1. A similar behavior is observed for the permeability constraint $(k)$, amplitude ratio $(\varepsilon)$ and angle of proclivity $(\theta)$ on the concentration profile.

2. An identical effect on the concentration profile is noticed for wall constraints ( $\left.E_{1}, E_{2}, E_{3}\right)$.

3. An opposite behavior of homogeneous response rate constraint $(\alpha)$ and heterogeneous response rate constraint $(\beta)$ are observed on the concentration profile.

4. Similar behavior noted for amplitude ratio $(\varepsilon)$ on dispersion coefficient.

5. The permeability constraint $(k)$, amplitude ratio $(\varepsilon)$ angle of proclivity $(\theta)$ and wall constraints $\left(E_{l}\right.$, $\left.E_{2}, E_{3}\right)$ support the dispersion but the homogeneous chemical response rate $(\alpha)$ and the heterogeneous chemical response rate $(\beta)$ resist the dispersion.

\section{Acknowledgements}

Authors are very much thankful to the reviewers, English language experts, editors and editorial team of the journal for their constructive suggestions to improve the manuscript quality.

\section{Nomenclature}

$a$ - amplitude of the wave

$C$ - viscous damping force coefficient

$\mathbb{C}$ - concentration of the substance

$c$ - velocity of the peristaltic wave

$D$ - diffusion coefficient of the chemical reaction

$D^{*}$ - equivalent dispersion coefficient

$d$ - half width of the channel

$\mathcal{E}_{1}$ - rigidity of the wall

$\mathcal{E}_{2}$ - stiffness of the wall

$\mathcal{E}_{3}$ - viscous damping force of the wall

$G$ - coefficient of scattering

$\bar{G}$ - average effective coefficient of scattering

$g$ - acceleration due to gravity

$\bar{k}$ - permeability constraint

$k_{1}$ - rate of chemical response

$m$ - mass per unit area

$L$ - motion of the stretched membrane

$p$ - pressure on the wall

$Q$ - volume flow rate

$T$ - tension in the membrane

$t$ - time

$\mathcal{U}, v$-velocity components in $x, y$ direction

$\alpha$-homogeneous response rate constraint

$\beta$-heterogeneous response rate constraint

$\gamma$ - gravitational constraint

$\varepsilon$-amplitude ratio

$\theta$ - angle of inclination of the channel

$\lambda$ - wavelength of the peristaltic wave

$\mu-$ coefficient of viscosity

$\rho-$ density of the fluid 


\section{References}

[1] Jaffrin M.Y., Shapiro A. and Weinberg H.S.L. (1969): Peristaltic pumping with long wavelengths at low Reynolds number. - Journal Fluid Mechanics, vol.37, pp.799-825.

[2] Fung Y.C. and Yih C.S. (1968): Peristaltic transport. ASME transactions. - Journal of Applied Mechanics, vol.35, No.4, pp.669-675.

[3] Misra J.C. and Ghosh S.K. (1997): A mathematical model for the study of blood flow through a channel with permeable walls. - Acta Mechanica, vol.122, pp.137-153.

[4] Seaway E.F. and El Sebaei W. (2000): Peristaltic transport in a cylindrical tube through a porous medium. International Journal of Mathematics and Mathematical Sciences. vol.24, pp.217-230.

[5] Takagi D. and Balmforth N.J. (2011): Peristaltic pumping of viscous fluid in an elastic tube. - Journal of Fluid mechanics, vol.672, pp.196-218.

[6] Meikhemeir Kh. S. (2003): Non-linear peristaltic transport of a second-order through a porous medium. Journal of Porous Media, vol.6, No.3, pp.189-201.

[7] Jayarami Reddy B., Subba Reddy M.V., Nadhamuni Reddy C. and Yogeswar Reddy P. (2012): Peristaltic flow of a Williamson fluid in an inclined planner channel under the effect of a magnetic field. - Advances in Applied Science research, vol.3, No.1, pp.452-461.

[8] Ali Ibrahim and Abdulhadi A.M. (2014): Peristaltic transport of a viscoelastic fluid with fractional Maxwell model in an inclined channel. - Qi Journal of Sciences, vol.4, No.B, pp.1962-1975.

[9] Kavitha A., Reddy H., Saravana R. and Sreenath R. (2015): Peristaltic transport of a Jeffrey fluid in contact with a Newtonian fluid in an inclined Channel. - Ain Sham Engineering Journal, http://dx.doi.org/10.1016/j.asej.2015.10.014.

[10] Mittra T.K. and Prasad N.S. (1973): On the influence of wall properties and poiseuille flow in peristalsis. Journal of Biomechanics, vol.6, pp.681-693.

[11] Sankad G.C. and Radhakrishnamacharya G. (2009): Effect of Magnetic Field on the peristaltic motion of micropolar fluid with wall effects. - Journal of Applied Mathematics and Fluid Mechanics, vol.1, pp.37-50.

[12] Ramana Kumari A.V. and Radhakrishnamacharya G. (2012): Effect of slip and magnetic field on peristaltic flow in an inclined channel with wall effects. - International Journal of Biomathematics, vol.5, N.6, http://dx.doi.org/10.1142/S1793524511001568.

[13] Sankad G.C. and Radhakrishnamacharya G. (2014): Influence of wall properties on the peristaltic transport of a micropolar fluid in an inclined channel. - Journal of New Results in Science, vol.6, pp.62-75.

[14] Rathod V.P., Manikrao N. and Sridhar N.G. (2015): Peristaltic flow of a couple stress fluid in an inclined channel under the effect of the magnetic field. - Pelagia Research Library, vol.6, No.9, pp.101-109.

[15] Hayat T., Tanveer A. and Alsaedi A. (2015): Simultaneous effects of radial magnetic field and wall properties on peristaltic flow of Carreau-Yasuda fluid in curved flow configuration. - AIP Advances, vol.5, No.12, http://dx.doi.org/10.1063/1.4939541.

[16] Riaz A., Ellahi R. and Nadeem S. (2014): Peristaltic transport of a Carreau fluid in a complaint rectangular duct. - Alexandria Engineering Journal, vol.53, pp.475-484.

[17] Ellahi R., Bhatti M.M., Fetecau C. and Vafai K. (2016): Peristaltic flow of couple stress fluid in a non-uniform rectangular duct having complaint walls. - Communications in Theoretical Physics, vol.65, No.1, pp.66-72.

[18] Taylor G.I. (1953): Dispersion of soluble matter in solvent flowing slowly through a tube. - Proceedings of Royal Society London, vol.219(A), pp.186-203.

[19] Aris R. (1956): On the dispersion of a solute in a fluid flowing through a tube. - Proceedings of Royal Society London, vol.35(A), pp.67-77. 
[20] Padma D. and Ramana Rao V.V. (1975): Homogeneous and heterogeneous reaction on the dispersion of a solute in MHD Couette flow - I. Current Science, vol.44, pp.803-804.

[21] Gupta P.S. and Gupta A.S. (1972): Effect of homogeneous and heterogeneous reactions on the dispersion of a solute in the laminar flow between two plates. - Proceedings of Royal Society London, vol.330(A), pp.59-63.

[22] Sobh A.M. (2013): Effect of homogeneous and heterogeneous reactions on the dispersion of a solute in MHD Newtonian fluid in an asymmetric channel with peristalsis. - British Journal of Mathematics \& Computer Science, vol.3, No.4, pp.664-679.

[23] Chandra P. and Philip D. (1993): Effect of heterogeneous and homogeneous reactions on the dispersion of a solute in simple microfluid. - Indian Journal of Pure Applied Mathematics, vol.24, pp.551-561.

[24] Dutta B.K.N., Roy N.C. and Gupta A.S. (1974): Dispersion of a solute in a non-Newtonian fluid with simultaneous chemical reaction. - Mathematica- Mechanica fasc., vol.2, pp.78-82.

[25] Alemayehu H. and Radhakrishnamacharya G. (2012): Dispersion of solute in peristaltic motion of a couple stress fluid through a porous medium. - Tamkang Journal of Mathematics, vol.43, No.4, pp.541-555.

[26] Hayat T., Tanveer A., Yasmin H. and Alsaedi A. (2014): Homogeneous-heterogeneous reactions in peristaltic flow with convective conditions. - PLOS one, vol.9, http://dx.doi.org/10.1371/journal.pone.0113851.

[27] Ravi Kiran G. and Radhakrishnamacharya G. (2015): Effect of homogeneous and heterogeneous chemical reactions of peristaltic transport of a Jeffrey fluid through a porous medium with slip condition. - Journal of Applied Fluid Mechanics, vol.8, No.3, pp.521-528.

[28] Gupta B.B. and Seshadri V. (1976): Peristaltic pumping in non-uniform tubes. - Journal of Biomechanics, vol.9, pp.105-109.

[29] Lightfoot E.N. (1974): Transport Phenomena in Living Systems. - New York: John Wiley and Sons.

Received: March 6, 2018

Revised: November 30, 2018 